\title{
Comparison of measured and simulated lateral distributions for electrons and muons with KASCADE
}

\author{
W.D. Apel ${ }^{\text {a }}$, A.F. Badea ${ }^{\mathrm{a}, 1}$, K. Bekk ${ }^{\mathrm{a}}$, A. Bercuci ${ }^{\mathrm{b}}$, \\ J. Blümer ${ }^{\text {a,c }}$, H. Bozdog ${ }^{\text {a }}$, I.M. Brancus ${ }^{b}$, A. Chilingarian ${ }^{d}$, \\ K. Daumiller ${ }^{\text {a }}$, P. Doll ${ }^{\text {a }}$, R. Engel ${ }^{a}$, J. Engler ${ }^{a}$, H.J. Gils ${ }^{a}$, \\ R. Glasstetter ${ }^{c, 2}$, A. Haungs ${ }^{a}$, D. Heck ${ }^{\mathrm{a}}$, J.R. Hörandel ${ }^{\mathrm{c}}$, \\ K.-H. Kampert ${ }^{c, a, 2}$, H.O. Klages ${ }^{a}$, G. Maier ${ }^{a, 3}$, H.J. Mathes ${ }^{\text {a }}$, \\ H.J. Mayer ${ }^{\text {a,*}}{ }^{\text {, J. Milke }}{ }^{\text {a }}$, M. Müller ${ }^{\text {a }}$, R. Obenland ${ }^{\text {a }}$, \\ J. Oehlschläger ${ }^{\mathrm{a}}$, S. Ostapchenko ${ }^{\mathrm{a}, 4}$, M. Petcu $^{\mathrm{b}}, \mathrm{H}$. Rebel ${ }^{\mathrm{a}}$, \\ A. Risse ${ }^{\mathrm{e}}$, M. Risse ${ }^{\mathrm{a}}$, M. Roth ${ }^{\mathrm{c}}$, G. Schatz ${ }^{\mathrm{a}}, \mathrm{H}$. Schieler $^{\mathrm{a}}$, \\ H. Ulrich ${ }^{a}$, J. van Buren ${ }^{a}$, A. Vardanyan ${ }^{d}$, A. Weindl ${ }^{a}$, \\ J. Wochele ${ }^{\mathrm{a}}$, J. Zabierowski ${ }^{\mathrm{e}}$ \\ a Institut für Kernphysik, Forschungszentrum Karlsruhe, 76021 Karlsruhe, \\ Germany \\ ${ }^{\mathrm{b}}$ National Institute of Physics and Nuclear Engineering, 7690 Bucharest, Romania \\ ${ }^{\mathrm{c}}$ Institut für Experimentelle Kernphysik, Universität Karlsruhe, 76021 Karlsruhe, \\ Germany \\ ${ }^{\mathrm{d} C o s m i c ~ R a y ~ D i v i s i o n, ~ Y e r e v a n ~ P h y s i c s ~ I n s t i t u t e, ~ Y e r e v a n ~ 36, ~ A r m e n i a ~}$ \\ e Soltan Institute for Nuclear Studies, 90950 Lodz, Poland
}

\begin{abstract}
Lateral distributions for electrons and muons in extensive air showers measured with the array of the KASCADE experiment are compared to results of simulations based on the high-energy hadronic interaction models QGSJet and SIBYLL. It is shown, that the muon distributions are well described by both models. Deviations are found for the electromagnetic component, where both models predict a steeper lateral shape than observed in the data. For both models the observed lateral shapes of the electron component indicate a transition from a light to a more heavy composition of the cosmic ray spectrum above the knee.
\end{abstract}




\section{Introduction}

The particle lateral distribution of extensive air showers (EAS) is the key quantity for cosmic ray ground observations, from which most shower observables are derived. The interaction cascade, which is initiated by a high energy cosmic ray particle in the atmosphere, creates a multitude of secondary particles, which arrive nearly at the same time but distributed over a large area perpendicular to the direction of the original particle. The disc of secondary particles may extend over several hundred meters from the shower axis, with maximum density in the center of the disc, which is called the shower core. Apart from the arrival times, the density distribution of particles within the shower disc contain all informations on the primary particle, which are left after it has undergone a millionfold multiplication process by the atmosphere. However, it is this multiplication process, that foremost offers the chance to observe cosmic rays in the ultra and very high energy region at all: Due to their low flux measurements at ground, carried out with large arrays of individual detectors which take samples of the shower disc at several locations, are still the only possible way to study these high-energy cosmic particles [1].

The lateral distributions of electrons and muons in EAS not only contains information on the nature of the primary cosmic ray particle, which is related to astrophysical questions, but it also carries information relevant to particle physics. While the electromagnetic interactions are thought to be well understood, this is not true for the high energy hadronic interactions. The energy range and the kinematical region in which the first hadronic interactions of the shower development occur are far beyond the accessible realm of todays accelerator experiments. Uncertainties in the description of hadronic interactions therefore imply uncertainties in the prediction of the shape of the lateral distributions [2].

A parameter, commonly used to describe the form of the lateral density distribution, is the lateral form parameter in the Nishimura-Kamata-Greisen (NKG)-function [3,4,5], usually called age. The name expresses the relation between the lateral shape of the electron distribution and the height of the shower maximum. Due to the statistical nature of shower development, the height of the shower maximum is subject to strong fluctuations. Showers,

\footnotetext{
* corresponding author, E-mail address: mayer@ik.fzk.de

1 on leave of absence from Nat. Inst. of Phys. and Nucl. Engineering, Bucharest, Romania

2 now at Fachbereich Physik, Universität Wuppertal, 42097 Wuppertal, Germany

3 present address: University of Leeds, Leeds LS2 9JT, UK

4 on leave of absence from Moscow State University, 119899 Moscow, Russia
} 
which have started high in the atmosphere show a flat lateral electron distribution, as electrons in the electromagnetic cascade suffer more from multiple scattering processes. Such showers are called old and are characterised by a large value of the age parameter. Young showers have started deeper in the atmosphere and had their maximum more close to observation level. This results in a steeper lateral electron distribution, which corresponds to a smaller value of the age parameter. Apart from fluctuations, the height of the shower maximum depends on energy and mass of the shower initiating primary. Therefore, the lateral shape parameter is also sensitive to the mass of the primary.

The mutual interrelation of several independent parameters on which the form of the lateral shape depends, makes it a delicate task to draw unique conclusions from the results of the measurements. Moreover, the interpretation of the measured raw data requires a profound understandig on the details of the detector response functions. Sophisticated simulations of the whole event chain that is initiated by the first collision of an ultrahigh energy cosmic ray particle with an air nucleus in the upper atmosphere and ends with the registration of electronical signals in the various detector components are a prerequisite to any reliable analysis of the lateral distributions of all particle components.

This view encourages to measure the secondary particle components separately, which from an experimental point of view requires several detector components to be operated simultaneously. The detector array of the multi detector setup KASCADE (Karlsruhe shower core and array detector) [6] is designed to disentangle the electromagnetic, the hadronic, and the muon component of the shower disc. Lateral distributions of electrons, hadrons, and muons (for different muon threshold energies) in the primary energy range $5 \cdot 10^{14} \mathrm{eV}<E<10^{17} \mathrm{eV}$ as measured with KASCADE have already presented in a previous paper [7]. In this paper, the lateral distributions of electrons and muons in EAS events as measured with the KASCADE array detectors will be compared with the predictions of detailed Monte Carlo calculations, which comprise the simulation of the full cascading process of EAS, the simulation of the array detector response and the final data reconstruction mechanisms. Whereas in [7] the parameterisations of the lateral distributions were analysed for mean values only, here the reconstruction is also performed on a single event basis. Special emphasis is given to investigations of the shape of the lateral distributions, the so-called 'lateral age', and its dependencies on primary energy and mass of the cosmic rays. Contrary to [7], the hadronic component, measured with the KASCADE central hadron calorimeter, will not be considered in the present analysis, as well as measurements from the additional KASCADE muon devices. 
The paper is organised as follows: After a brief description of the experimental setup an overview on the simulation methods is given. Then we shortly outline the data reconstruction scheme. A more detailed explanation is given on the method we use for the reconstruction of electron numbers and on function used to describe the measured and simulated lateral shapes. This is followed by the presentation of mean lateral distributions for muons and electrons, as measured with the KASCADE array and a comparison with the results from the simulations. Then we show the results for the lateral shape of individual showers and its dependence on the shower observables electron and muon number. The simulations results, the data will be compared with, are mostly based on the hadronic interaction model QGSJet [8], but simulations with lower statistics based on the SIBYLL model [9] have also been performed. Therefore, results based on the SIBYLL model and the differences in the predictions of both models are discussed at the end of the paper.

\section{The KASCADE experiment}

The KASCADE experiment is located at the site of the Forschungszentrum Karlsruhe at an altitude of $110 \mathrm{~m}$ above sea level. A central hadron calorimeter is surrounded by a rectangular array of 252 scintillation detector stations, equally spaced by $13 \mathrm{~m}$ and covering an area of $200 \mathrm{x} 200 \mathrm{~m}^{2}$. In addition, there is a muon tracking detector with an effective area of $128 \mathrm{~m}^{2}$. The experiment measures the hadronic, muonic and electromagnetic components of extensive air showers in the energy range of $5 \cdot 10^{14} \mathrm{eV}$ up to $10^{17} \mathrm{eV}$ of the primary particles. A detailed description of the experiment can be found in [6].

The 252 detector stations of the KASCADE detector array are organized in 16 electronically independent clusters. Each cluster consists of 16 stations, except the inner four clusters, where one station per cluster had to give way to the central detector. The stations of the inner four clusters contain four liquid scintillation detectors, each with an area of $0.8 \mathrm{~m}^{2}$ read out by one photomultiplier. The stations of the outer clusters contain two such detectors with $1.6 \mathrm{~m}^{2}$ total area. All photomultiplier signals of a detector station are added, and the integrated charge of the signal is recorded, together with the time of the earliest detector hit by a shower particle. These detectors are designed to measure arrival time and energy deposits of the electromagnetic component of the showers and are therefore referred to as $e / \gamma$-detectors here.

Additionally, the stations of the 12 outer clusters house $3.2 \mathrm{~m}^{2}$ plastic scintillation detectors below a shielding of $10 \mathrm{~cm}$ lead and $4 \mathrm{~cm}$ of iron, which gives $0.23 \mathrm{GeV}$ threshold for muons. Each detector is read out by 4 photomultiplier 
devices and in turn yields time and energy deposit information. Again, the sum of the multiplier signals is recorded together with the hit pattern and the time of the earliest detector hit. These detectors measure the muon component and are referred to as muon-detectors here.

The shower observables which are reconstructed with the KASCADE array data are core position, shower direction and the lateral distribution of electrons and muons. From this, the shower size, expressed as total number of electrons $N_{e}$ above $3 \mathrm{MeV}$ and a lateral shape parameter will be derived. For the muon component only the total number of muons $N_{\mu}$ above $100 \mathrm{MeV}$ can be estimated. Due to the low muon densities, a reliable determination of the lateral shape parameter is in general not possible for single event analysis.

\section{Monte Carlo simulation}

A reliable interpretation of the data requires a detailed understanding of the physics of shower development, as well as a detailed knowledge of the detector response. The whole event chain, starting with the primary interaction in the upper atmosphere, followed by the cascading of the shower particles through the air up to the response of the detectors at KASCADE ground level has been simulated carefully.

The simulation of extensive air showers is performed with the program CORSIKA (version 6.156 and higher) [10]. For the high energy hadronic interactions, the models QGSJet (version 01) [8] and SIBYLL (version 2.1) [9] are used. Hadronic interactions with energies below $200 \mathrm{GeV}$ are treated with the FLUKA code [11], and the electromagnetic component is treated with the EGS4 package [12]. The showers were simulated in the energy range from $1 \cdot 10^{14} \mathrm{eV}$ to $1 \cdot 10^{17} \mathrm{eV}$. With respect to computing time the distribution in energy was chosen to follow a power law with spectral index $\gamma=-2$. To represent different primary masses, the set contains equal numbers of showers for five different primary types, namely protons, helium, carbon, silicon and iron. The position of the shower cores were distributed randomly all over the array. For the shower directions an isotropic distribution was chosen. Output of the program is a list of all particles reaching KASCADE ground level together with their coordinates, arrival time, 3-momenta and particle type.

These data are input to the KASCADE simulation program, which is based on the GEANT3 package [13]. The simulation covers the whole experiment, with all its detectors modelled in great detail. All particles from a CORSIKA 
simulated shower are tracked through the detectors, the surrounding air and the absorber materials. Secondaries, created in interactions with the detector materials are likewise followed. In case of the array stations, energy deposits and timing information are gathered during the tracking step and converted into a photomultiplier signal and a signal time taking into account the light collecting geometry of the detector as well as the specific properties of the scintillation material. Output of this program, concerning the array part, are arrival times and multiplier signals for $e / \gamma$ - and muon-detectors in exactly the same format, that is written by the real experiment after the calibration procedure, i.e. the simulated data can be analysed with the standard KASCADE reconstruction software. A more detailed description of the KASCADE array simulation can be found in [14].

\section{Reconstruction of particle density distributions and shower pa- rameters}

Detector simulations applied to CORSIKA shower events have also been extensively used for the development and testing of the array data reconstruction algorithms and procedures. As the KASCADE array reconstruction scheme has already been described in several previous papers $[6,7,14]$, only a brief overview will be given in this chapter. Those parts of the analysis chain, which are concerned with the reconstruction of the lateral electron distribution and its properties will be described in more detail, as they were subject to modifications applied for the present analysis.

\subsection{Reconstruction of the $e / \gamma$ component}

Shower direction and shower core position, as well as shower size and the lateral form parameter (usually known as age parameter) are reconstructed from energy deposits and detector response times of the $e / \gamma$-detectors using an iterative procedure involving three steps.

In the first step a rough estimate of the shower direction, core position and shower size is obtained using fast and robust algorithms, which don't rely on any fit procedure. In the second step, the shower direction is determined more accurately, by evaluating the arrival times of the first particle in each detector. This yields an inclination resolution better than 0.3 degrees for showers with $\lg N_{e}>4.5$ [6]. Then, corrections depending on core position and shower inclination are applied to the individual detector energy deposits. From this, particle numbers and corresponding particle densities are calculated for each 
detector. A 4-parameter fit to the spatial distribution of the particle densities yields core position and shower size, and in addition, a lateral shape parameter of the charged particle density distribution. The core position resolution at this level of reconstruction is better than $0.3 \mathrm{~m}$ for showers with $\lg N_{e}>4.5$, the shower size resolution at the percentage level [6]. The e/ $\gamma$-detector signals contain contributions also from the muon component, for which must be corrected for, which is performed in the third step of the reconstruction scheme: As the analysis of the muon-detector data proceeds in parallel to the analysis of the e/ $\gamma$-detector data, the total muon number $N_{\mu}$ is known at the time of step three from the step two muon analysis. Therefore, the expected muon density can be estimated individually for each e/ $\gamma$-detector. The resulting signal contribution is then accounted for in the detector probabilities for the Likelihood minimisation function and the combined lateral density distributions are fitted. Since the accuracy in core position is in general not further improved in this step, only shower size and lateral form parameter are varied, yielding the final values of the total electron number $N_{e}$, and the shape parameter of the lateral electron density distribution.

\subsubsection{Reconstruction of particle densities}

Reconstruction of the lateral particle density distribution requires to interprete the measured energy deposits in terms of particle numbers. After correcting for different track lengths in the scintillator due to shower inclination, special attention must be paid to the $\gamma$-component. The electrons are accompanied by a multitude of $\gamma$-particles, which fake additional electrons, because the photon efficiency of the scintillation detectors is roughly $10 \%$. The percentage of fake electrons strongly depends on core distance, because the mean $\gamma / e$-ratio is a function of core distance. In addition, the detector efficiency for electrons decreases with increasing core distance, as their kinetic energy distribution becomes more and more soft with growing core distance. Moreover, both effects depend on shower size and primary particle type.

A shower size dependent lateral energy correction function (LECF) has been derived using the Monte Carlo simulations described in chapter 3. This function gives the average expected energy deposit per shower electron as a function of core distance and shower size and thereby accounts for the additional deposit due to accompanying photons and the dependence of the detector efficiency on the kinetic energy of the electrons. Dividing the measured energy deposit by the expected deposit per shower electron, an estimate on the number of electrons, hitting the detector is given.

Figure 1 left shows LECFs for proton induced showers for different shower sizes. The figure also shows the parametrisation of the mean $\gamma / e$-ratio $q_{\gamma / e}$ 

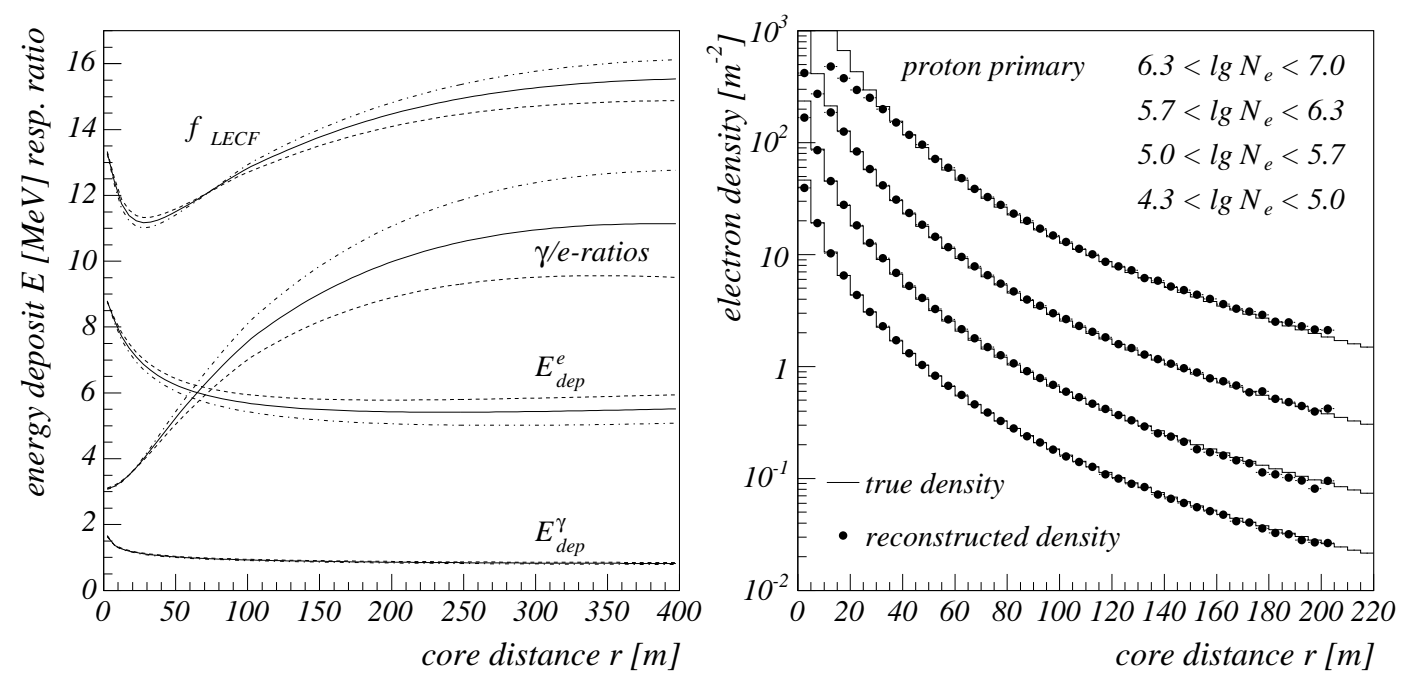

Fig. 1. Left: Proton LECF's for $\lg N_{e}=5$ (dashed lines), $\lg N_{e}=6$ (full lines) and $\lg N_{e}=7$ (dashed dotted lines). Also shown are corresponding $\gamma /$ e-ratios and mean energy deposits for electrons and photons as parametrised and used for the LECF. Right: Mean electron densities as reconstructed from simulated detector data for proton induced CORSIKA showers. The solid lines give the original CORSIKA electron densities. Only electrons and photons have been tracked through the detectors in this case.

and the mean energy deposits $E_{d e p}^{e}, E_{d e p}^{\gamma}$ per electron and photon, respectively. The energy $E_{d e p}^{t o t}$ deposited in average by $n_{e}$ electrons and $n_{\gamma}$ accompanying photons in the detector is then given by

$$
E_{d e p}^{t o t}=n_{e} \cdot E_{d e p}^{e}+n_{\gamma} \cdot E_{d e p}^{\gamma}
$$

From this the functional form of the LECF ist derived according to

$$
f_{L E C F} \equiv \frac{E_{d e p}^{t o t}}{n_{e}}=E_{d e p}^{e}+\frac{n_{\gamma}}{n_{e}} \cdot E_{d e p}^{\gamma} \approx E_{d e p}^{e}+q_{\gamma / e} \cdot E_{d e p}^{\gamma}
$$

where the mean electron and photon energy deposits are assumed to depend only on the mean kinetic particle energies.

The sudden fall of the LECF starting at the shower center reflects the decrease of the mean kinetic energy of electrons and $\gamma$ 's with increasing core distance. At a core distance of about $30 \mathrm{~m}$, the corresponding loss in detector efficiency becomes compensated by the rising $\gamma$-electron ratio, which yields an increasing fraction of fake deposit due to $\gamma$-particles. At large core distances, $\gamma$ 's fake nearly half one of the average energy deposit per electron.

Comparing LECFs calculated for proton and iron primaries, one finds them to 
differ by less than $1 \%$ within $100 \mathrm{~m}$ from the shower core. For larger distances, the differences do not exceed $5 \%$. So one is free to use a common LECF for the analysis of data, where the primary particle type is unknown. Moreover, the variation with shower size is less than $100 \mathrm{~m}$. There is a negligible dependence also on the inclination of the showers, as geometrical effects are corrected for in the reconstruction procedures.

The right part of Figure 1 shows results of the reconstruction when applied to simulated detector deposits, calculated from CORSIKA showers with the detector Monte Carlo, as described above. To check the reliability of the LECF, only electrons and $\gamma$-particles have been tracked in this case. The reconstructed electron distributions compare well to the distributions of the CORSIKA electrons. Deviations from the original distribution are found only for large showers and small core distance. This however is due to saturation effects in the $e / \gamma$-detectors, which are included in the detector simulation.

\subsubsection{Reconstruction of shower parameters size and lateral shape}

A theoretically motivated function for the description of the lateral electron density distribution $\rho(r)$ is given by the so-called Nishimura-Kamata-Greisen function $(\mathrm{NKG})[3,4,5]$

$$
\rho=N_{e} \cdot c(s) \cdot\left(\frac{r}{r_{M}}\right)^{s-2}\left(1+\frac{r}{r_{M}}\right)^{s-4.5}
$$

with the age parameter $s$, the Moliere radius $r_{M}$ and the normalising factor $c(s)$

$$
c(s)=\frac{\Gamma(4.5-s)}{2 \pi r_{M}^{2} \Gamma(s) \Gamma(4.5-2 s)} .
$$

This function has been derived analytically for the case of purely $e / \gamma$-induced air showers but is also used to describe the lateral electron distribution of hadron induced showers. It is however known $[15,16,17]$, that the NKG functions have shortcomings in fitting measured EAS electron distribution, most obvious at large core distances. This deficit is usually addressed to the fact, that the NKG-function was derived for electromagnetic cascades, whereas hadron induced air showers are a superposition of a large number of independent electromagnetic showers.

In a typical KASCADE event, the detector distances to the core may extend up to $200 \mathrm{~m}$. In case of large showers with sizes well above $\lg N_{e}=6$, which 

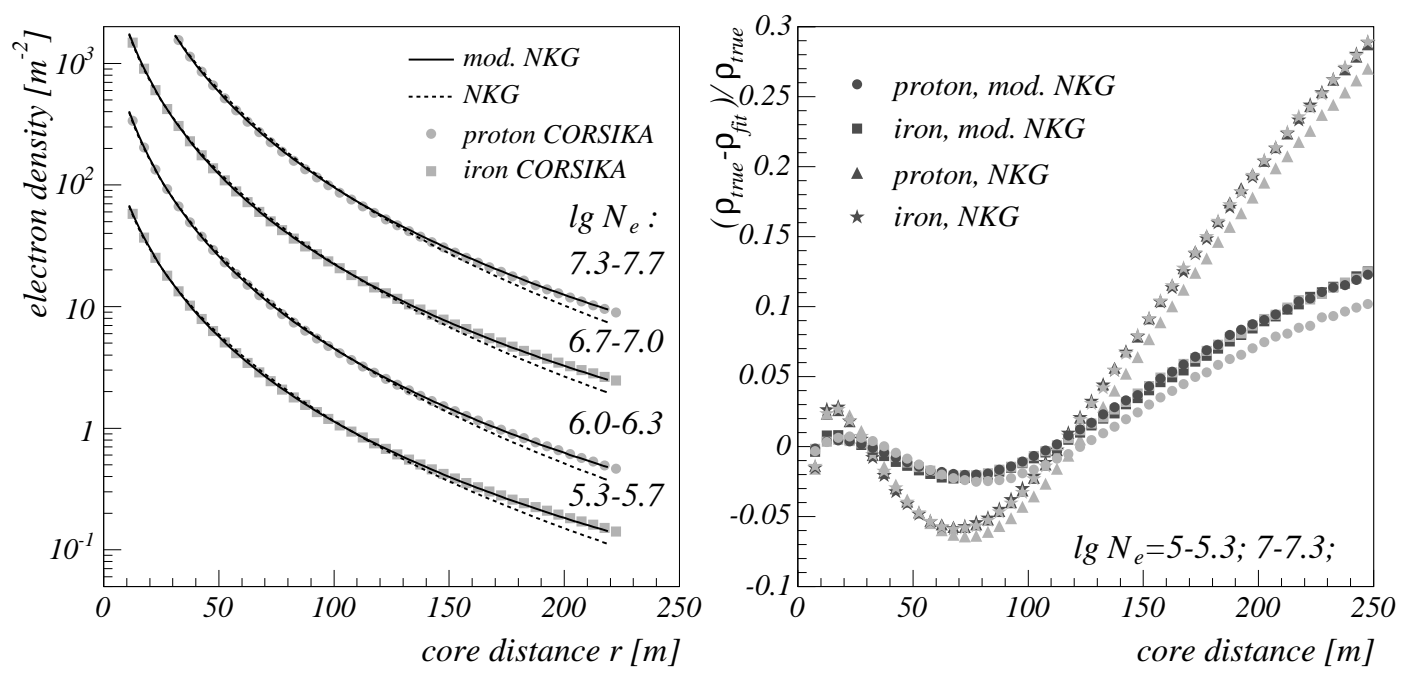

Fig. 2. Left: Comparison of fit results for the standard NKG function and the modified NKG function. Shown are, as an example, fitted CORSIKA electron distributions (i.e. without detector simulation) for proton and iron showers and two different shower sizes each. Right: Residuals of the fitted function and the simulated CORSIKA electron distributions for proton and iron induced showers and two different shower sizes (dark symbols represent the small, grey symbols the large $N_{e^{-}}$bin). The iron symbols mostly overlap.

roughly correspond to a primary energy of $10 \mathrm{PeV}$, the detectors close to the shower core become saturated and must be rejected for the analysis. Thus, the lateral fit range differs for small and for large showers significantly in both, the upper and lower bound. The deviation of the NKG-function from the true shape of the lateral electron distribution therefore gives rise to systematic errors in the lateral shape parameter (age $s$ in the NKG-formula), which depend on the lateral fit range, and thereby on shower size.

It has been pointed out [7], that fixing the age parameter $s$ and instead varying the scale parameter $r_{0}$ (Moliere radius $r_{M}$ in the NKG-formula) considerably improves the fit behaviour of the NKG function. Unfortunately, this does work well only for mean lateral distributions constructed from a large number of showers. When fitting individual events, which suffer from large statistical and physical fluctuations between the detector stations, this method has proven to be significantly unstable. Especially for small showers it yields unreliable results.

Other ways to cure the defects of the NKG function for describing electron lateral distributions of hadron induced air showers, is to replace it by a different function (e.g. as in [18]) or to modify its functional form by changing the values of the exponents. Indeed, this gives a better adaption to the shape of the lateral 
density distribution. For this, we replace equation (2) by

$$
\rho=N_{e} \cdot \tilde{c}(s) \cdot\left(\frac{r}{r_{0}}\right)^{s-\alpha}\left(1+\frac{r}{r_{0}}\right)^{s-\beta}
$$

with

$$
\tilde{c}(s)=\frac{\Gamma(\beta-s)}{2 \pi r_{0}^{2} \Gamma(s-\alpha+2) \Gamma(\alpha+\beta-2 s-2)} .
$$

Testing this function with Monte Carlo data, as optimum values for the exponents we have found $\alpha=1.5$ and $\beta=3.6$, when $r_{0}=40 \mathrm{~m}$ for the scale parameter is used. Optimum in this case means an almost negligible systematic uncertainty in the reconstructed shower size $N_{e}$ over the full KASCADE range as shown in Figure 3. With these values of $\alpha, \beta$ and $r_{0}$, equation (5) limits the new parameter $s$ to the range $-0.5<s<1.55$. At the same time, of course, it looses its numerical relation to the longitudinal development of the electromagnetic cascade, as it is often mentioned for its original form. In the following, the new parameter $s$ will be called the shape or form parameter of the lateral density distribution.

As an example, the left part of Figure 2 compares both variants of the fit function when applied to mean lateral electron distributions derived directly (i.e. without detector simulation) from CORSIKA simulated proton and iron showers for two different shower sizes. The modified NKG-function adapts much better to the lateral shapes, which becomes most obvious at large core distances. This can be seen even better at the right part of Figure 2, which compares relative deviations of the fit function from the distributions for the original NKG-function and the modified one. Apart from the very vicinity of the shower core, the modified NKG-function describes the shape of the mean lateral distributions with significantly smaller residuals over the whole fit range.

The benefits of the modified NKG-function when applied to individual showers are shown in Figure 3. The left part shows results for the estimate of the total shower size, derived by both functions. With the modified function, the systematic uncertainty in shower size is almost zero over the range $5<\lg N_{e}<7$ and still below $5 \%$ between $4.5<\lg N_{e}<7.5$. Even more convincing, this does not depend on the primary particle type, contrary to the results from the original NKG function, which fits iron induced shower profiles with a larger systematic uncertainty than proton induced ones. 

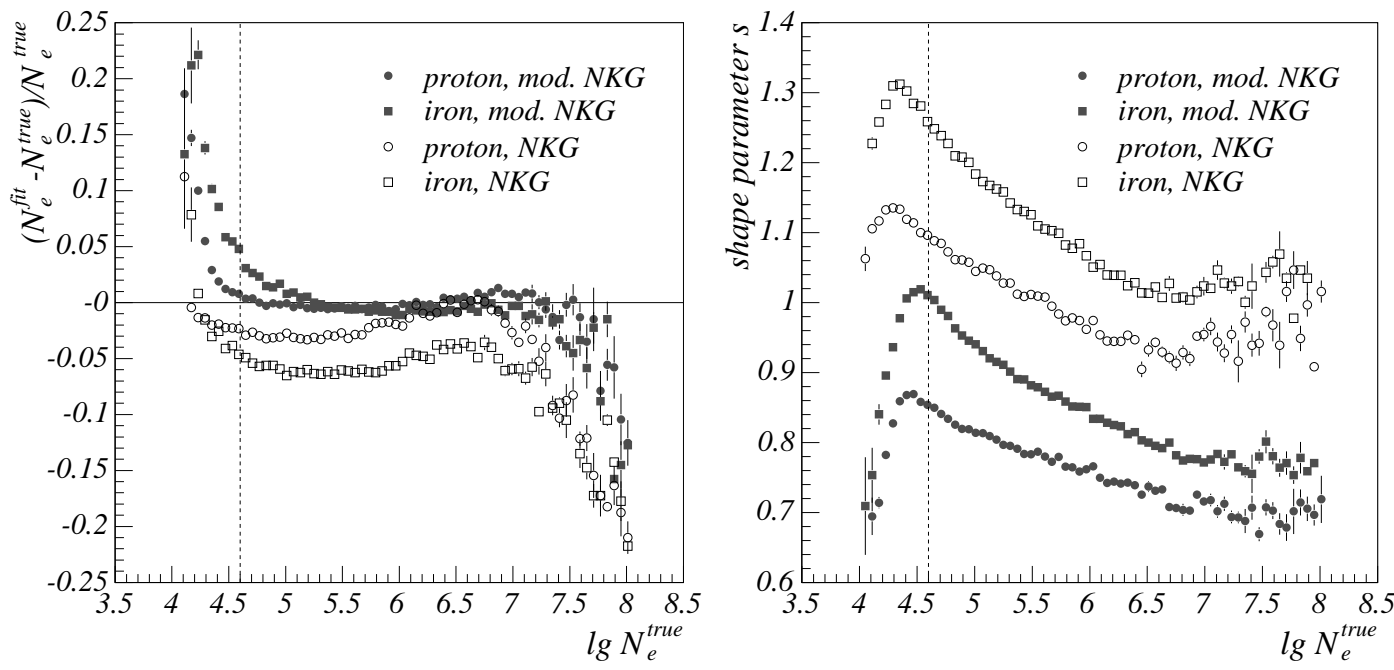

Fig. 3. Left: Deviation of reconstructed from true shower size for NKG and modified NKG function, when fitting individual showers. Right: Reconstructed shape parameter as a function of shower size using NKG and modified NKG function. The rise of the age parameter for showers with $\lg N_{e}>6.5$ is an artefact of the shortcomings of the NKG function in describing the lateral distributions at large core distance. The vertical dashed lines shows the KASCADE threshold.

The increasing systematic error towards small shower sizes below $\lg N_{e}=5$ in the case of iron primaries is related to the strongly growing $\mu / e$-ratio. For $\lg N_{e}=4$, the muon density becomes comparable to the electron density even at small core distances. This makes it difficult to disentangle both components in the $e / \gamma$-detector analysis and finally leads to an overestimate in shower size. For $\lg N_{e}>7$ on the other hand, more and more detectors in the vicinity of the shower core get saturated. This reduces the available lateral fit range and results in a quickly growing underestimate of the shower size. Both effects ultimately establish the limits in the primary energy range for this analysis to the region between $5 \cdot 10^{14} \mathrm{eV}$ and $10^{17} \mathrm{eV}$.

The right part of Figure 3 compares the results of the original and the modified NKG function for the lateral shape parameter as a function of the reconstructed shower size. As already mentioned, the absolute value of the shape parameter is related to the choice of the scale radius $r_{0}$ and the values taken for the exponents $\alpha$ and $\beta$ of the modified NKG-function and is therefore shifted to smaller values. Apart from that, the results of the conventional fit function exhibit a rise of the shape parameter for $\lg N_{e}>6.6$ due to the described shortcomings of the original NKG-function and the shrinking of the lateral fit range with growing shower size, when detectors near to the shower core become saturated. This artefact is absent with the new fit function. For shower sizes below the KASCADE threshold the age distributions are effected by the selection procedures. 


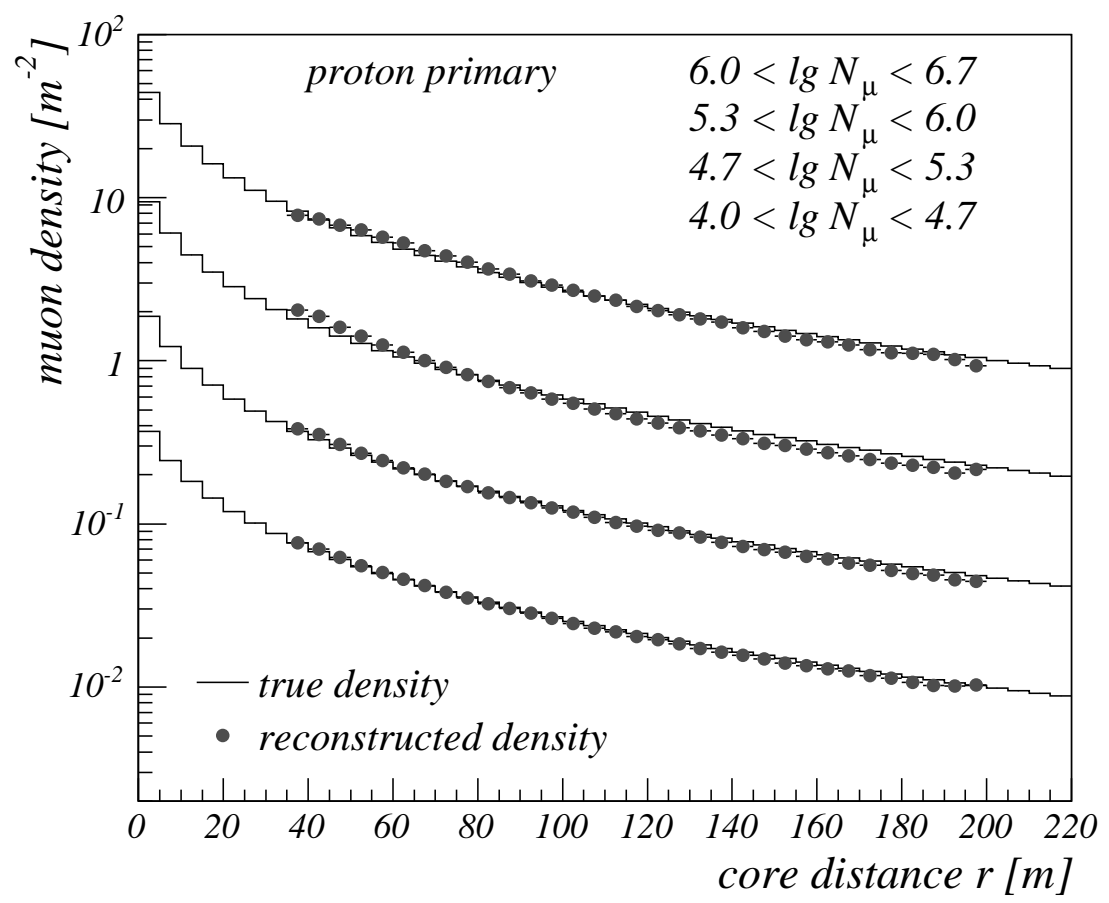

Fig. 4. Muon lateral densities as reconstructed from simulated muon-detector data compared to the true CORSIKA densities.

\subsection{Reconstruction of the $\mu$-component}

The analysis of the muon-detector signals follows the same line as in the case of the e/ $\gamma$-detectors. Again, the raw signals are subject to corrections due to shower inclination and core position using a corresponding muon LECF, which has a much simpler form than in the $\mathrm{e} / \gamma$-case. Additionally, corrections for punch through from the $\mathrm{e} / \gamma$ - and hadron component are applied before the muon densities for every detector are calculated. Detectors closer than $40 \mathrm{~m}$ to the shower core must be excluded from the analysis, because in this region punch through dominates the signal.

The total muon number is estimated by fitting a modified NKG function with exponents $\alpha=1.5$ and $\beta=3.7$. For the muon component this gives only a moderate improve over the original NKG-function, which is known to fit muon lateral distributions already quite well, provided the scale parameter is chosen appropriately. Here we take $r_{0 \mu}=420 \mathrm{~m}$. Due to the low muon densities, a 2-parameter fit on a single air shower basis proves unreliable. Therefore the total muon number $N_{\mu}$ of the shower is estimated with a fixed lateral form parameter $s_{\mu}$ and a 1-parameter fit. The muon lateral shape $s_{\mu}$ is parametrized as a function of shower size $N_{e}$ from CORSIKA simulations and the actual 
value is chosen event by event during the iterative reconstruction. For the considered data sample $s_{\mu}$ varies between 0.81 and 0.75 , slowly decreasing with increasing shower size.

Reconstruction results for muon densities are illustrated in Figure 4, where muon distributions are compared for simulated showers with the corresponding true CORSIKA distributions for several ranges of the reconstructed total muon number $N_{\mu}$. For individual showers, the accuracy of this observable is moderate compared with shower size $N_{e}$ and is not better than 10 to 20\%. This results from the poor muon statistics in case of small showers and punch through at large shower sizes. The muon size $N_{\mu}$ is input to the correction of shower size and shape parameter in the third step of the $\mathrm{e} / \gamma$-detector analysis.

\section{Comparisons of KASCADE data with Monte Carlo simulations}

For the results presented here all measured showers with $\log N_{e}>6$ have been taken into account. This sample comprises about 170000 events measured in a period of nearly 8 years. Additionally, these data have been enriched with a sample of the many small showers, which hit the array more frequently due to the steep energy spectrum of cosmic rays. For this, two KASCADE runs with in total about 2.5 million recorded shower events were added to the data set.

All showers, real or simulated, included in this analysis were subject to the same reconstruction procedure and to the same cuts concerning trigger condition, core position, inclination angle and shower shape parameter. Showers are restricted to core distances less than $90 \mathrm{~m}$ from the array center and zenith angles less than 30 degrees. An additional cut for showers with shape parameter value $s>1.4$, which is close to the upper boundary of the mathematically possible range, excludes showers which are frequently misreconstructed inside the array but actually had its core outside, or are just very small showers which fluctuated in such a way that the reconstruction overestimated their size by a large amount. Indeed, showers of the second kind are already very efficiently excluded, by comparing shower sizes as reconstructed during step one and step two of the analysis, and cutting on those events, where the difference in the estimated sizes exceeds the expectations due to the uncertainties in both methods considerably. Since the energy distribution of the simulated showers represents a spectral index of $\gamma=-2$ while the real data follow an index with $-2.6<\gamma<-3.1$ fluctuations to larger values in shower size would be less pronounced in simulations. Therefore appropriate statistical weights have been given to the simulated events, as will be explained below. 
The analysed KASCADE data set is first compared to the predictions of the QGSJet model, based on a sample of 1.7 Million simulated events. In addition a set of showers with half that statistics but based on the SIBYLL model was analysed (section 5.4).

\subsection{Lateral distributions of muons}

Figure 5 shows mean lateral distributions from the simulations based on the QGSJet model and compares them with the data. Showers have been sampled in ranges of the reconstructed total muon number per single event. Because of the steeper energy spectrum of the data, fluctuations in muon number would give significantly larger contributions to higher $N_{\mu}$-bins than they would for simulated events. To account for this effect and also to show its result on the form of the lateral distributions, the simulated events have been analysed for a statistical weight representing a spectral index $\gamma=-2.6$ as well as for weights giving an index $\gamma=-3.2$. The spectral index of the data varies with energy, but will lie somewhere between these values. The small shaded bands in Figure 5 give the simulation results within this bounds. The lower bound of the band always corresponds to the larger absolute index value, i.e. to $\gamma=-3.2$. The width of the band for an individual primary mass is in the order of the symbol size, only. It is obvious, that the form of the lateral distribution function does only weakly depend on primary energy. Moreover, it can be seen, that the bands of proton and iron nuclei at least partially overlap. The simulations therefore predict, that the form of the muon distribution is not sensitive to the nature of the primary particle. At low energies, proton induced showers show slightly steeper lateral shapes compared to iron primaries, but these differences vanish for higher energies. Even for small showers the differences in the density distributions for showers of either type do not exceed ten percent.

The lateral distributions derived from the data agree quite well with the Monte Carlo predictions. The simulated distributions describe the measurements over the full KASCADE range of core distances and primary energies. The figure thereby shows clearly, why the muon measurements at KASCADE are sensitive to the primary energy, but give no valuable information on the elemental composition of cosmic rays. This is also found by using the SIBYLL model.

\subsection{Lateral distributions of charged particles}

Figure 6 presents lateral distributions of charged particles as measured by the $\mathrm{e} / \gamma$-detectors together with distributions derived from simulations. Though the bulk of particles are electrons, also muons contribute to the e/ $\gamma$-detector 


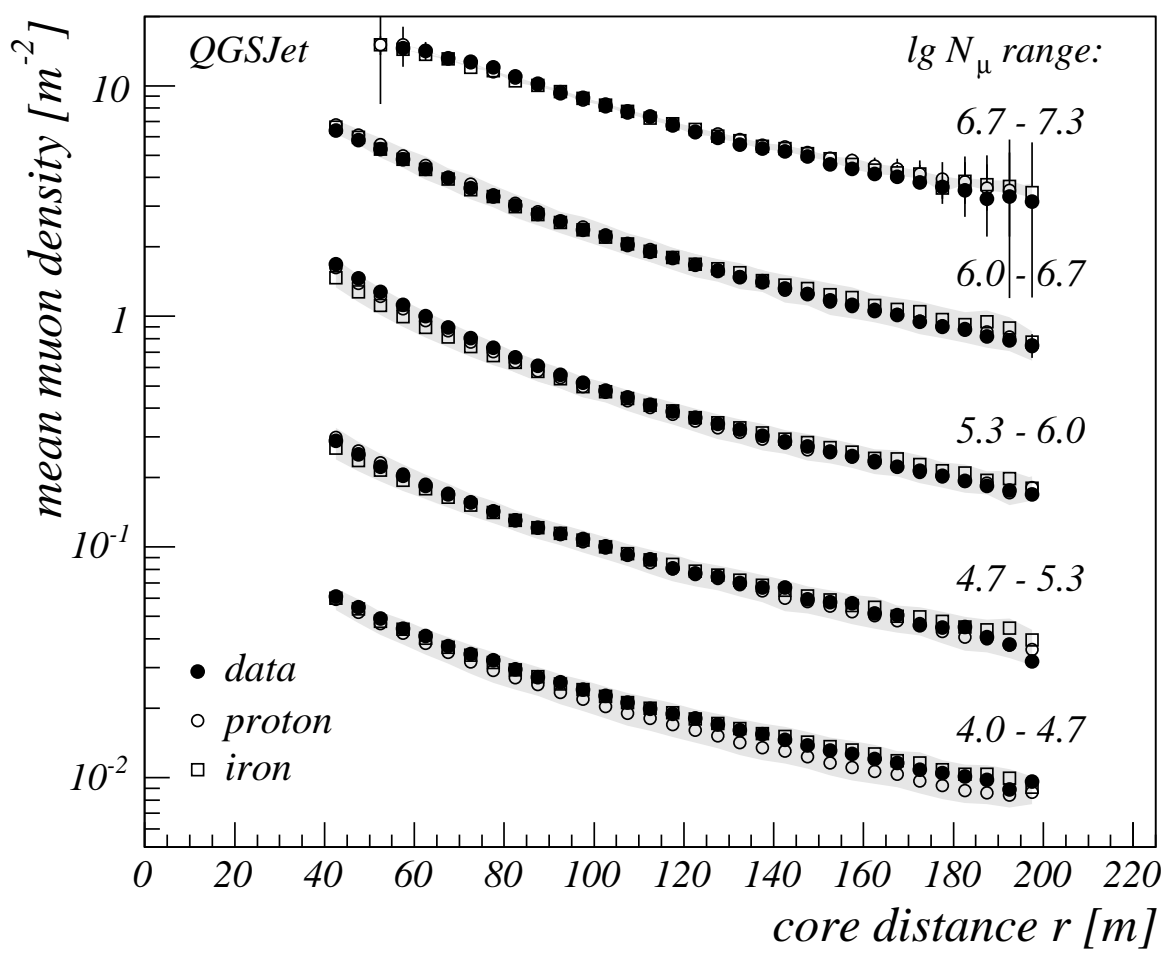

Fig. 5. Lateral density distributions of muons as measured with the KASCADE array muon-detectors. The shaded bands cover the range of the Monte Carlo simulation results with respect to five different elemental masses including an uncertainty in the spectral index within the range $-3.2<\gamma<-2.6$. For reasons of clarity, only the results for two elemental masses are shown additionally. For these, a spectral index $\gamma=-3$ is assumed.

signals, while contributions of photons are corrected by the LECF, and contaminations by hadrons are negligible. Therefore, the distributions presented here contain besides electrons also muons. All showers have been grouped according to their reconstructed total muon number.

The shaded bands of Figure 6 again indicate the range of uncertainty, which results, when the spectral index is varied between $-3.2<\gamma<-2.6$ and the primary mass from proton to iron. Again, the lower bounds corresponds to the larger absolute value $\gamma=-3.2$, the upper bounds to $\gamma=-2.6$. In addition, within each band the lateral distributions for five different primary masses are shown, assuming a spectral index of $\gamma=-3$ for each. The curve close to the upper bound of each band now results from the lightest primary, the one close to the lower bound belongs to iron. This expresses the well known fact [19], that showers originating from light primaries are more electron rich at sea level than showers induced by heavy nuclei. The $e / \mu$-ratio is therefore a common starting point for the analysis of the chemical composition of cosmic rays. Furthermore, the figure illustrates, that showers induced by light pri- 


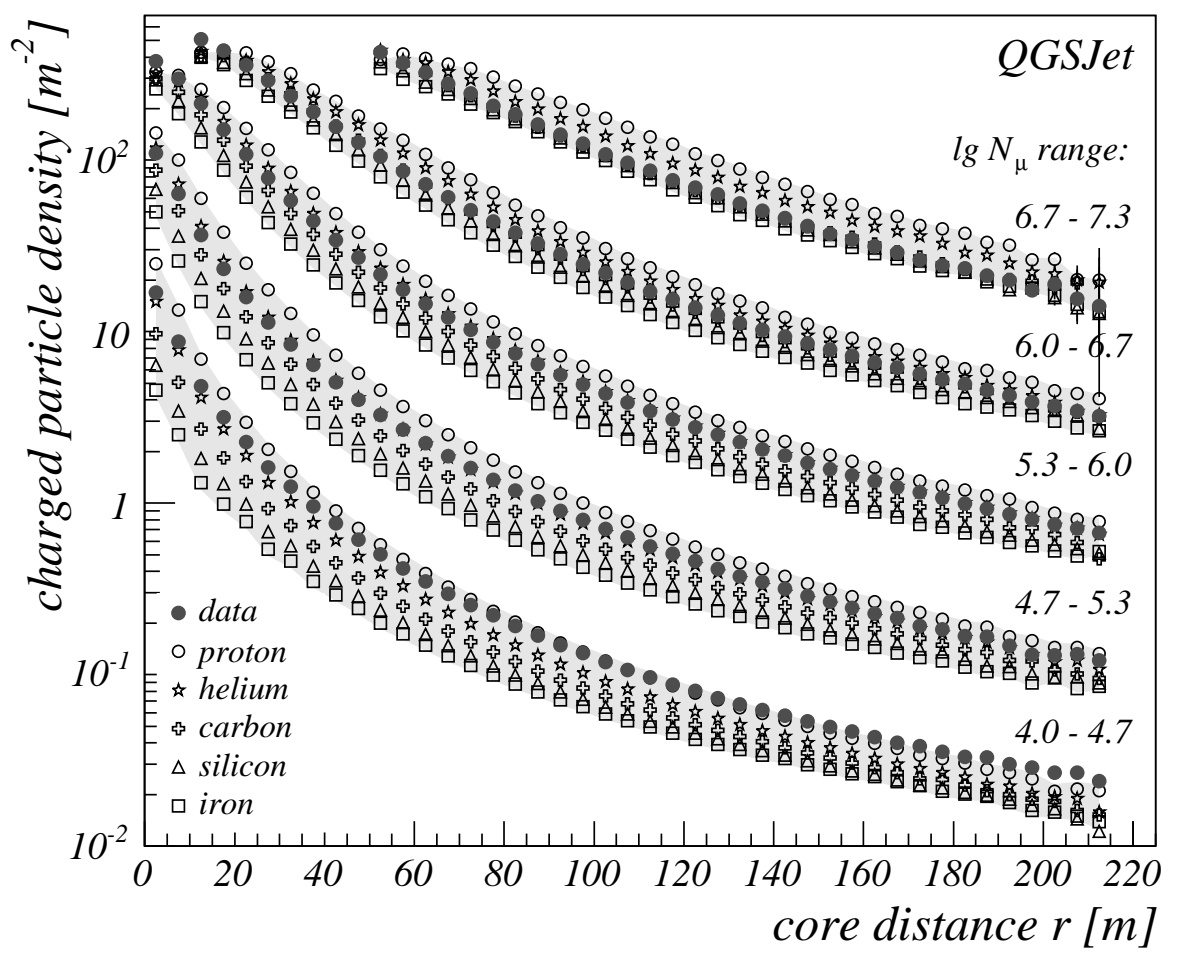

Fig. 6. Lateral density distributions of electrons including muons as measured with the KASCADE $e / \gamma$-detectors and by simulations. The shaded bands cover the range of the simulation results with respect to five different elemental masses including an uncertainty in the spectral index within the range $-3.2<\gamma<-2.6$. The results for each elemental mass, which are also shown, assume a spectral index $\gamma=-3$.

maries are predicted to exhibit a slightly steeper electron distribution than showers stemming from heavy nuclei.

Comparing real data with simulations, one sees that small showers with $\lg N_{\mu} \approx$ 4 fit simulated proton and helium distributions quite well, while large showers with $\lg N_{\mu} \approx 7$ are best described by the silicon and iron distributions, i.e. by heavier primary particles. The figure visualizes the known $[6,20]$ variation in the average $e / \mu$-ratio with shower size, which indicates, within the scope of the QGSJet model, a transition of the primary particle composition from light elements at energies below to heavy elements at energies above the knee. A detailed analysis of the electron-muon-number frequency spectrum as measured by KASCADE is subject of a separate paper, focused on the chemical composition of cosmic rays [21].

A closer look to Figure 6 also reveals discrepancies between the data and the QGSJet Monte Carlo predictions. At low energies, i.e. small muon numbers, 
the shapes of the measured distributions are slightly flatter than those of the simulated proton and helium distributions. For the smallest showers considered here, the measured particle densities even exceed the expected range of densities at core distances beyond $120 \mathrm{~m}$. This kind of shortcoming can not be cured by any assumption on the elemental composition and will be discussed in more detail in the next chapter.

Looking at the highest energies, where data are best described by heavy primary showers, simulations seem to show slightly lower densities and a flatter lateral behaviour at small core distances. In this region however, data suffer severely from overflows, from which additional uncertainties result.

It might be worth mentioning, that the shortcomings described here must originate completely from processes involved in the generation and development of the $e / \gamma$-component. The muon component contributes at low shower energies considerably to the lateral distributions measured with the $e / \gamma$-detectors. But the form of the muon lateral distribution is well described by the Monte Carlo simulations and therefore can not be drawn to explain the observed deviations.

\subsection{The lateral shape parameter}

The most obvious differences between the lateral shapes of the individual elements shown in Figure 6 are simply the amplitudes of the density functions, and are related to different $e / \mu$-ratios. A more subtle quantity, which in this kind of representation is difficult to compare, concerns the functional form or the slope of the lateral distribution.

The relations of shower sizes $N_{e}$ and $N_{\mu}$ with the shape parameter $s$ and with the primary mass are illustrated by Figure 7 . It shows the mean reconstructed shape parameter value as a function of the observables $\lg N_{e}$ and $\lg N_{\mu}$ for QGSJet based simulated showers. The individual events are weighted in energy to represent an elemental composition according to the results for the QGS modell as described in [21]. The lines overlaid to the distribution represent linear approximations to lines of maximum probability for showers of a single element (here shown only for proton and iron) but variable energy. It is obvious, that showers from light primaries are younger in average, i.e. have smaller shape values compared to heavy primaries, and showers of high energy are younger than low energy ones. 


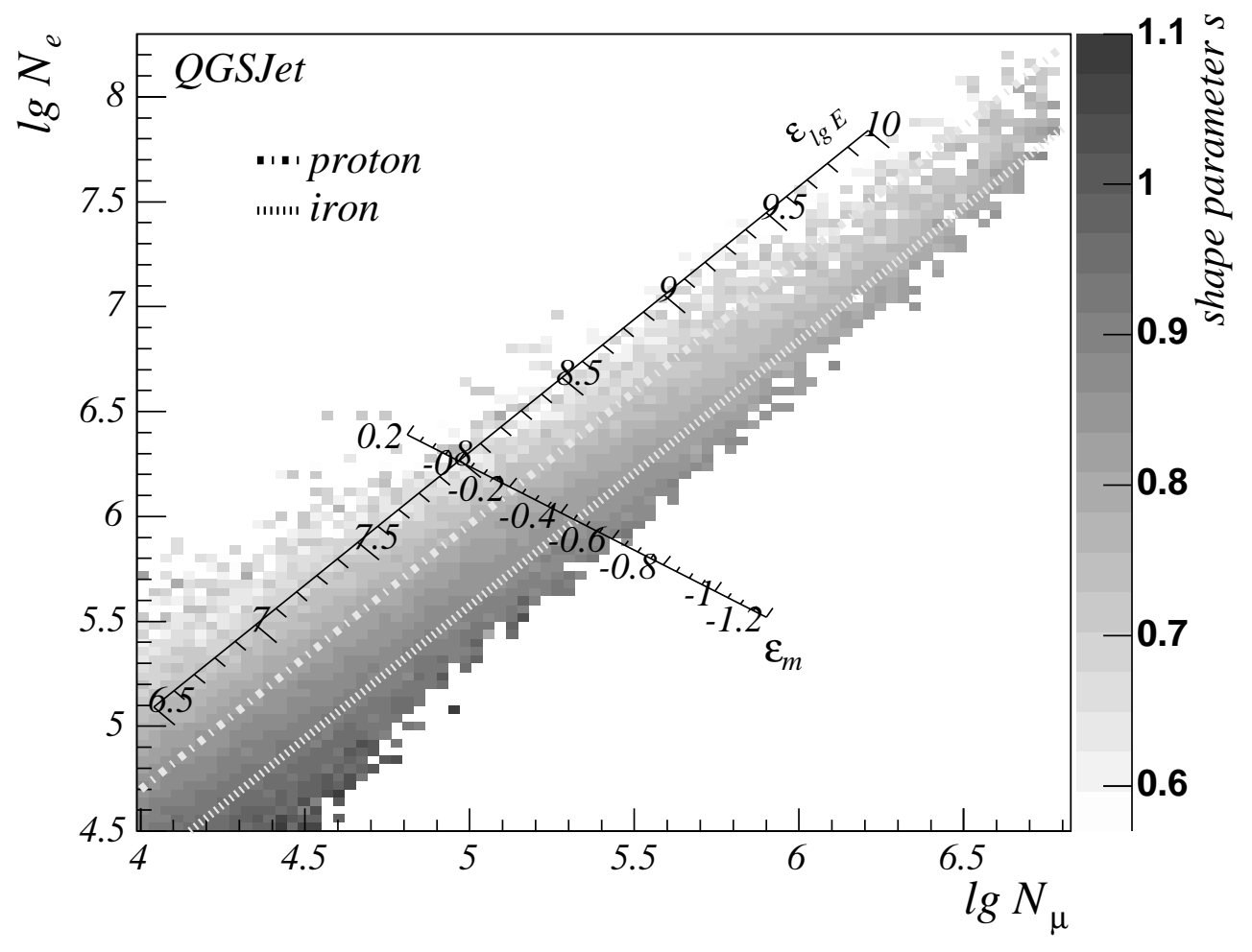

Fig. 7. Mean shower shape parameter $s$ as a function of $\lg N_{\mu}$ and $\lg N_{e}$ for simulated showers based on the QGSJet model with a composition adopted from [21]. Also shown are the lines of maximum probability for proton and iron induced showers and the coordinate system, used for the comparison of simulation results and data.

The lines of maximum probability in Figure 7 offer one axis of a natural, rectangular chosen coordinate system to compare the data with the simulation results. The new coordinates are related to the $\lg N_{\mu}-\lg N_{e}-$ system by a simple rotation around the origin with an angle of 51,6 degrees, obtained from the simulations. This coordinate system simply adapts the form of the event distribution in the $\lg N_{\mu}-\lg N_{e}$ - plane and will be used in the following to compare data and Monte Carlo results only on the basis of measured (or simulated) observables. While the coordinates along the lines of maximum probability may be associated with an energy estimator $\varepsilon_{\lg E}$, the coordinates perpendicular to this direction measure a mass estimator $\varepsilon_{m}$. These are surely not the best possible estimators for energy and mass but we do not want to draw quantitative conclusions from them. Therefore the numerical values of the new coordinates are simply the values obtained by the rotation from the old $\lg N_{\mu}-\lg N_{e}$-values.

The shape parameter as a function of the energy estimator $\varepsilon_{\lg E}$ is shown in Figure 8 for both, data and Monte Carlo simulations of all five elements. The Monte Carlo results confirm that showers induced by heavy primaries are older 


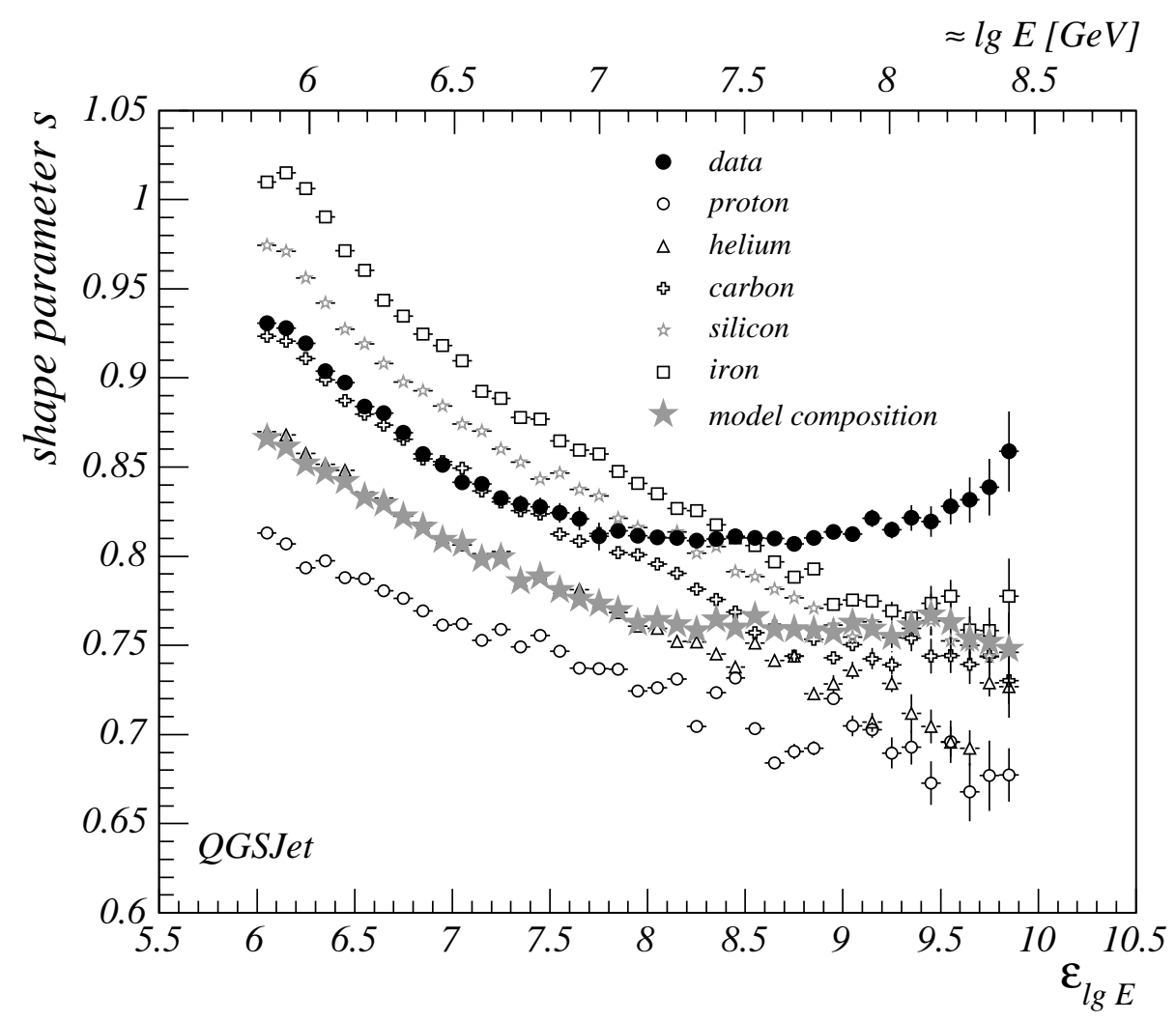

Fig. 8. Reconstructed shape parameter (of the modified NKG function) as a function of the energy estimator $\varepsilon_{\lg E}$ for KASCADE data, five primary masses and a model composition as simulated using QGSJet. The scale on top gives a rough estimate for $\lg E$ in $\mathrm{GeV}$.

compared to showers of light primaries. With increasing energy the shape parameter value decreases for all simulated elements and reflects the fact, that the height of the shower maximum decreases with increasing energy.

The data fit into this picture only qualitatively. Up to an energy of about $10 \mathrm{PeV}$, they follow the line of carbon. For low energies, this suggests a relatively heavy composition, which clearly disagrees with the predictions of figure 6. For higher energies, the lateral shape parameter stays almost constant and crosses the line of iron at an energy of about $30 \mathrm{PeV}$. Beyond this crossing point, the absolute values of the measured shape parameter cannot be explained by any elemental composition within this Monte Carlo model.

For a more detailed investigation the data distributions are compared with what would be expected from the simulations, once a reasonable elemental composition is given. For this, the simulated shower events of the five elemental masses have been weighted with individual energy spectra, which have been reconstructed from an analysis of the measured $N_{e} / N_{\mu}$-spectrum using 

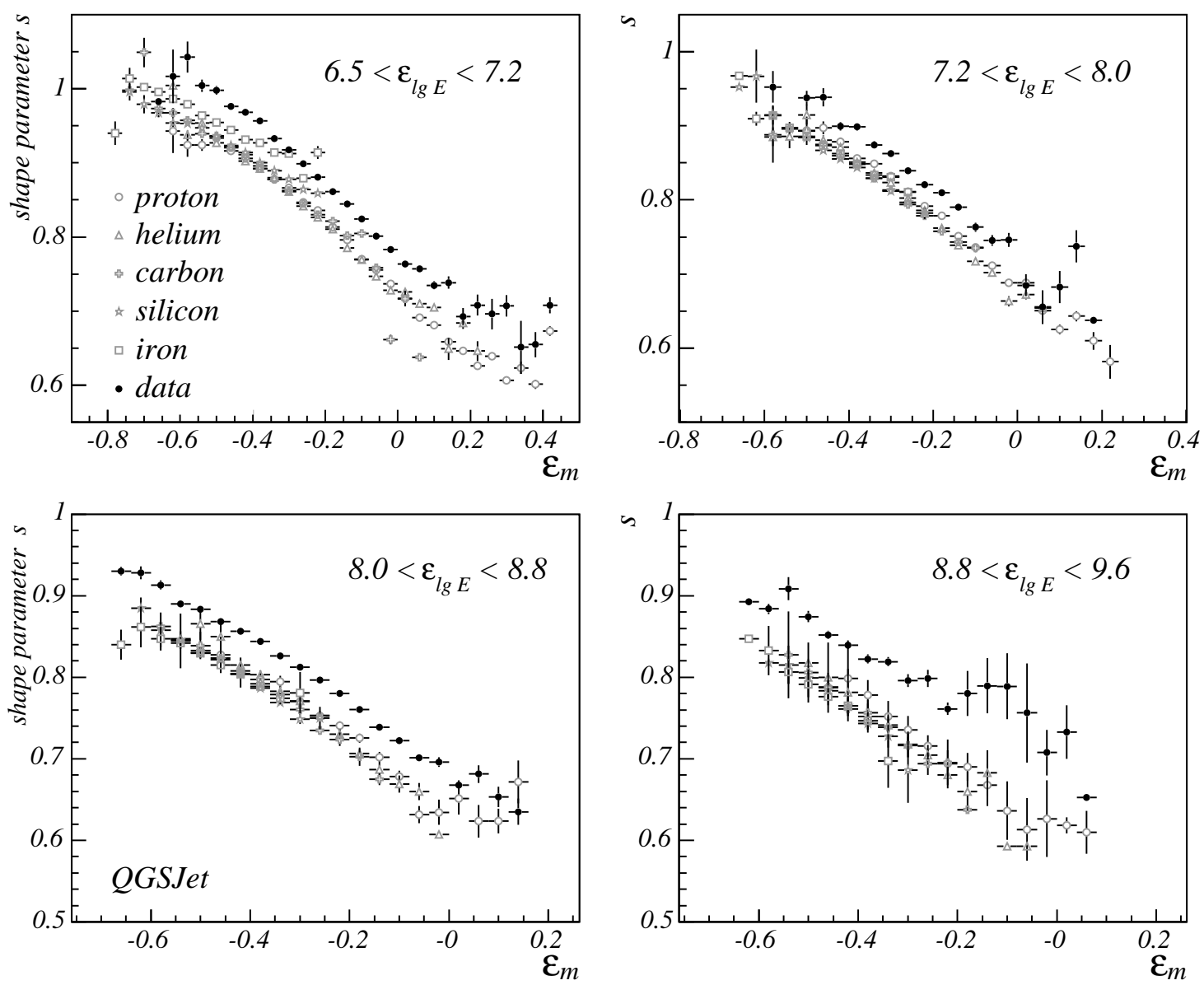

Fig. 9. Reconstructed shape parameter (of the modified NKG function) as a function of the mass estimator $\varepsilon_{m}$ for KASCADE data and five primary masses as simulated using QGSJet for different ranges of the energy estimator $\varepsilon_{\lg E}$. The line of maximum probability for protons corresponds to a value $\varepsilon_{m}=-0.22$ and for iron it is $\varepsilon_{m}=-0.45$.

a sophisticated unfolding algorithm based on the same model QGSJet. The resulting model composition favours light elements before the knee and a significant contribution from heavy elements at energies above the knee [21]. The effect on the shape parameter as a function of the energy estimator is also shown in Figure 8. It is remarkable, that the line of the measured shape parameter values runs almost parallel to the line representing these adapted Monte Carlo predictions, but is displaced by a nearly constant amount of $\Delta s \sim 0.05$ over the whole energy range.

The behaviour shown in Figure 8 may therefore be interpreted in the same way as the form of the lateral shapes in Figure 6 . The almost constant value of the shape parameter for energies beyond $10 \mathrm{PeV}$ can be understood as the result of a transition from light to heavy nuclei in the elemental composition of cosmic rays. The offset between the lines of measured and simulated shape 
simply states, that the simulations in general yield slightly steeper shapes than observed in real showers.

This can be seen even more clearly when looking along the lines of constant values of the mass estimator $\varepsilon_{m}$. This view is given in Figure 9 for several ranges of the energy estimator $\varepsilon_{\lg E}$, i.e. slices of Figure 7 perpendicular to the lines of maximum probability. Here higher mass values correspond to smaller shower size and larger muon content, i.e. to showers which are electron-poorer. Remarkably, all elemental masses can be seen to follow the same (energy dependent) functional dependence between the shape parameter and the mass estimator. On the other hand this may be expected, because showers of heavy primaries develop higher in the atmosphere compared to showers of light nuclei but same energy. However, the total number of electrons present in the shower maximum is, for showers of the same energy, roughly independent of the kind of the primary nucleus. Therefore, a deeply penetrating iron shower may not be distinguishable in shape from a proton shower, which developed very early in the atmosphere. The shape of real showers however does not follow this functional dependence. Measured showers are older in average, with values deviating by an amount $\Delta s \sim 0.05$ from simulated showers, with increasing tendency at the highest energies. This might indicate, that real showers develop at higher altitudes than predicted by the simulations or/and that multiple coulomb scattering plays a distinct and more pronounced role in the development of the real electromagnetic cascade expected from simulations.

\subsection{Comparison with the SIBYLL model}

The data have also been compared with simulations based on the SIBYLL model. While the $e / \mu$-ratio for SIBYLL showers is larger in general, no notable differences to the QGSJet model were found when comparing the shapes of the lateral distributions of muons for equal total muon numbers. The lateral shapes of the electron component show only small differences compared to the QGSJet model, as can be seen from Figures 10 and 6. The SIBYLL calculated shapes predict a more heavy composition, as a result of small differences in the $e / \mu$-ratios. In addition, the mean lateral electron distributions appear a bit younger.

Investigating the dependence of the lateral form parameter on the energy estimator as done in section 5.3, one can see in Figure 11 that SIBYLL describes the data worse compared to QGSJet. The SIBYLL iron curve crosses the data already at an energy of about $10 \mathrm{PeV}$, so there is no explanation for the measured shape values within this model for larger energies. Comparing with Figure 8 one finds that the mean shape of SIBYLL showers in general is 


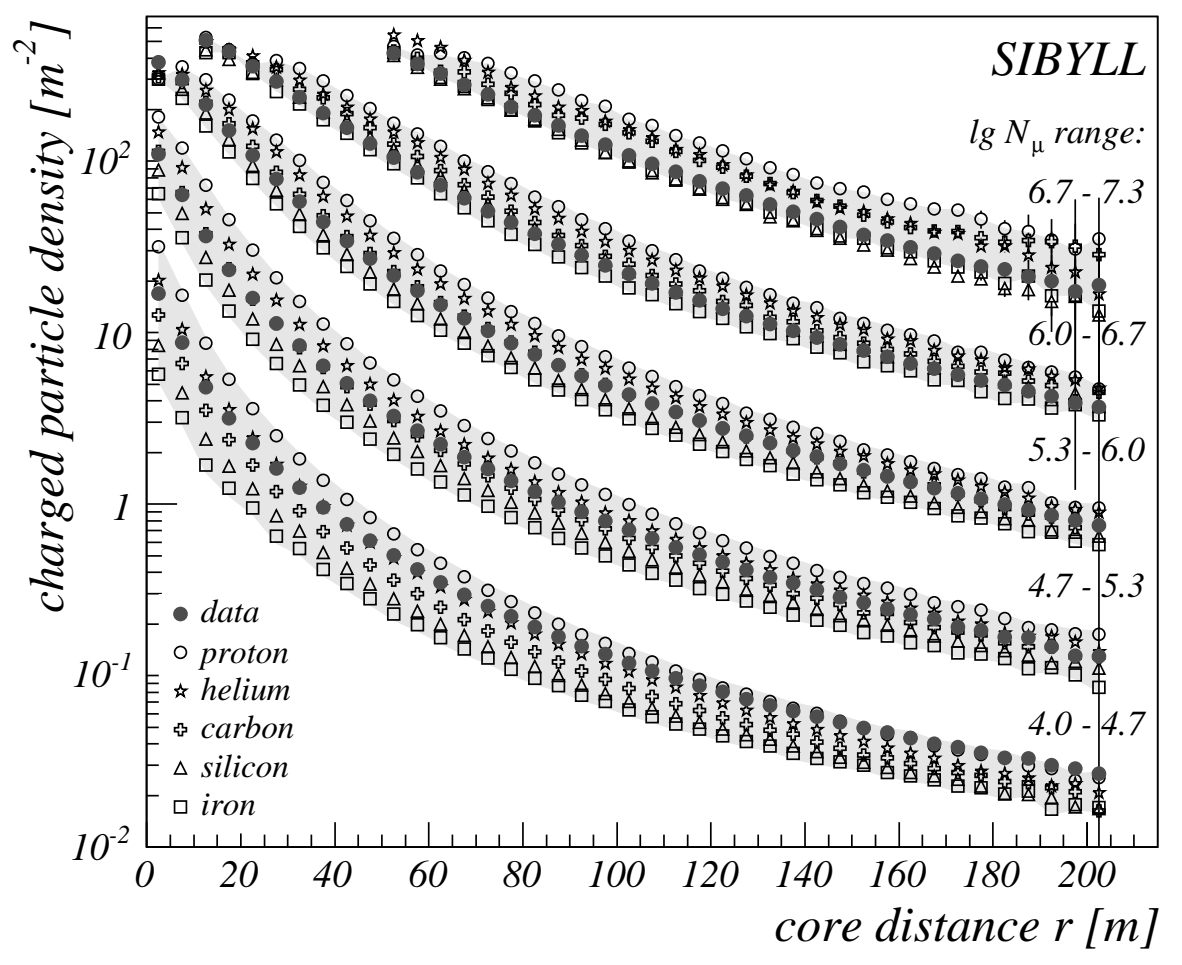

Fig. 10. Same as Figure 6, but with SIBYLL generated showers.

smaller by $\Delta s \sim 0.05$ compared to QGSJet.

It may appear surprising then, that individual SIBYLL showers follow the same functional dependence on the mass and energy estimators (and therefore also on $\lg N_{e}$ and $\lg N_{\mu}$ ) than QGSJet showers do, as can be seen from Figure 12. This shows that the longitudinal development of the electromagnetic component must be very similar in both models. The difference in the mean lateral shape parameter is therefore simply due to a different distribution of SIBYLL events in the $N_{e}-N_{\mu}$-plane (see also [21]). SIBYLL showers are more electron rich and produce less muons. The abundancy maximum for a given primary energy is therefore shifted to lighter mass values, enhancing the weight of younger showers when averaging on the shape parameter.

\section{Summary and conclusions}

Lateral electron and muon density distributions of air showers as measured with the KASCADE array have been compared to the results of Monte Carlo simulations based on the CORSIKA program using EGS4 and the two hadronic 


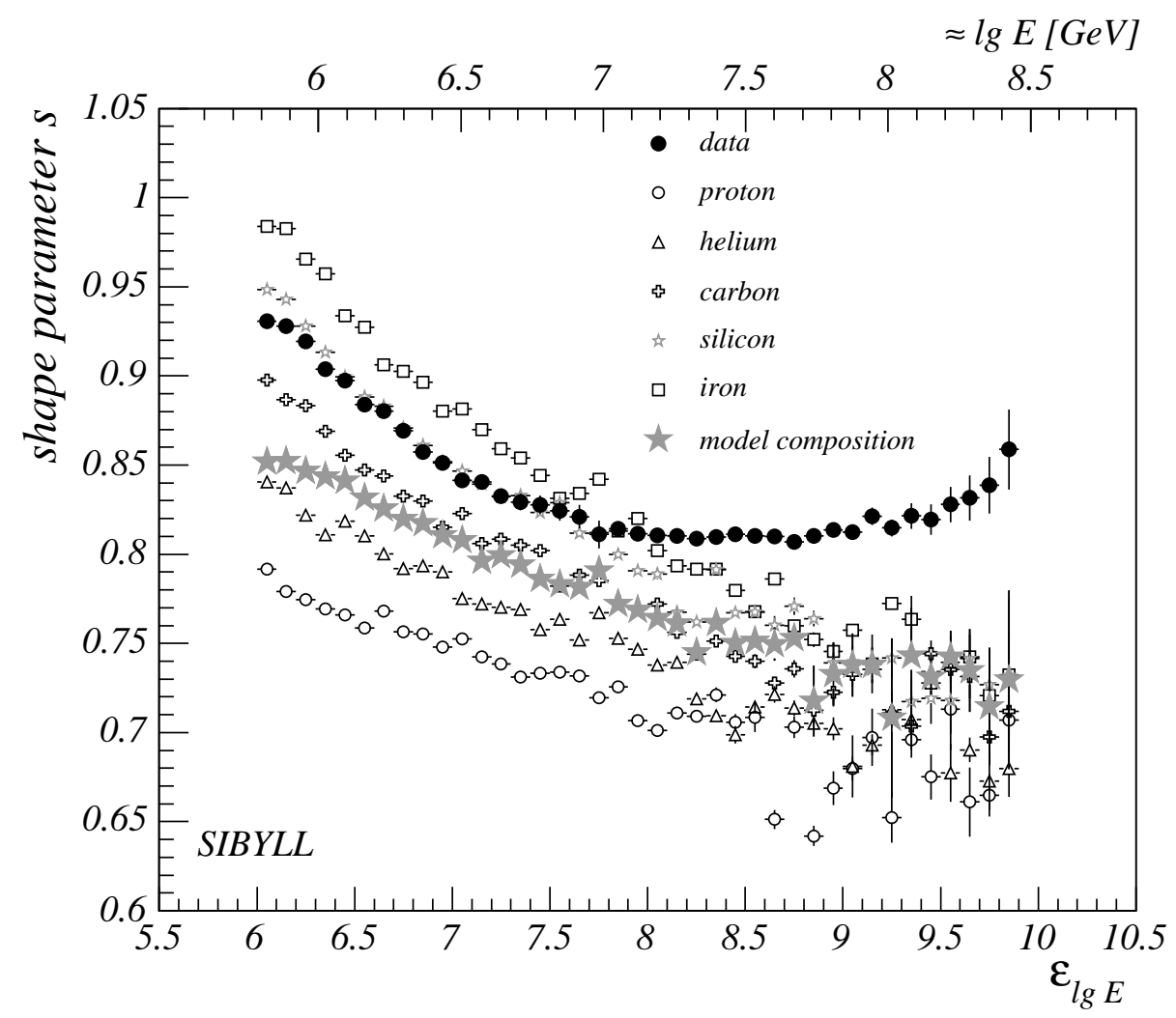

Fig. 11. Same as Figure 8, but with SIBYLL generated showers.

interaction models QGSJet and SIBYLL.

Muon lateral distributions measured with the KASCADE array muon detectors were found to be well described by the Monte Carlo simulations and no significant differences were observed between the two hadronic interaction models QGSJet and SIBYLL. Moreover, muon lateral distributions appear very similar in shape, independent of the nature of the primary particle, so that details of the chemical composition can not show up in the comparison of data and simulations.

Deviations from the Monte Carlo predictions are found for the lateral distributions of charged particles, which were reconstructed from the measurements of the KASCADE e/ $\gamma$-detector array. Common to both models is that they suggest a transition from light to heavy nuclei in the chemical composition of cosmic rays in the energy range of $1 \mathrm{PeV}$ to $100 \mathrm{PeV}$. This is consistent with the results of an independent study [21] based on a detailed analysis of the $\lg N_{e}-\lg N_{\mu}-$ frequency spectrum of KASCADE events.

Investigating in detail the shape of the lateral distributions, the absolute val- 

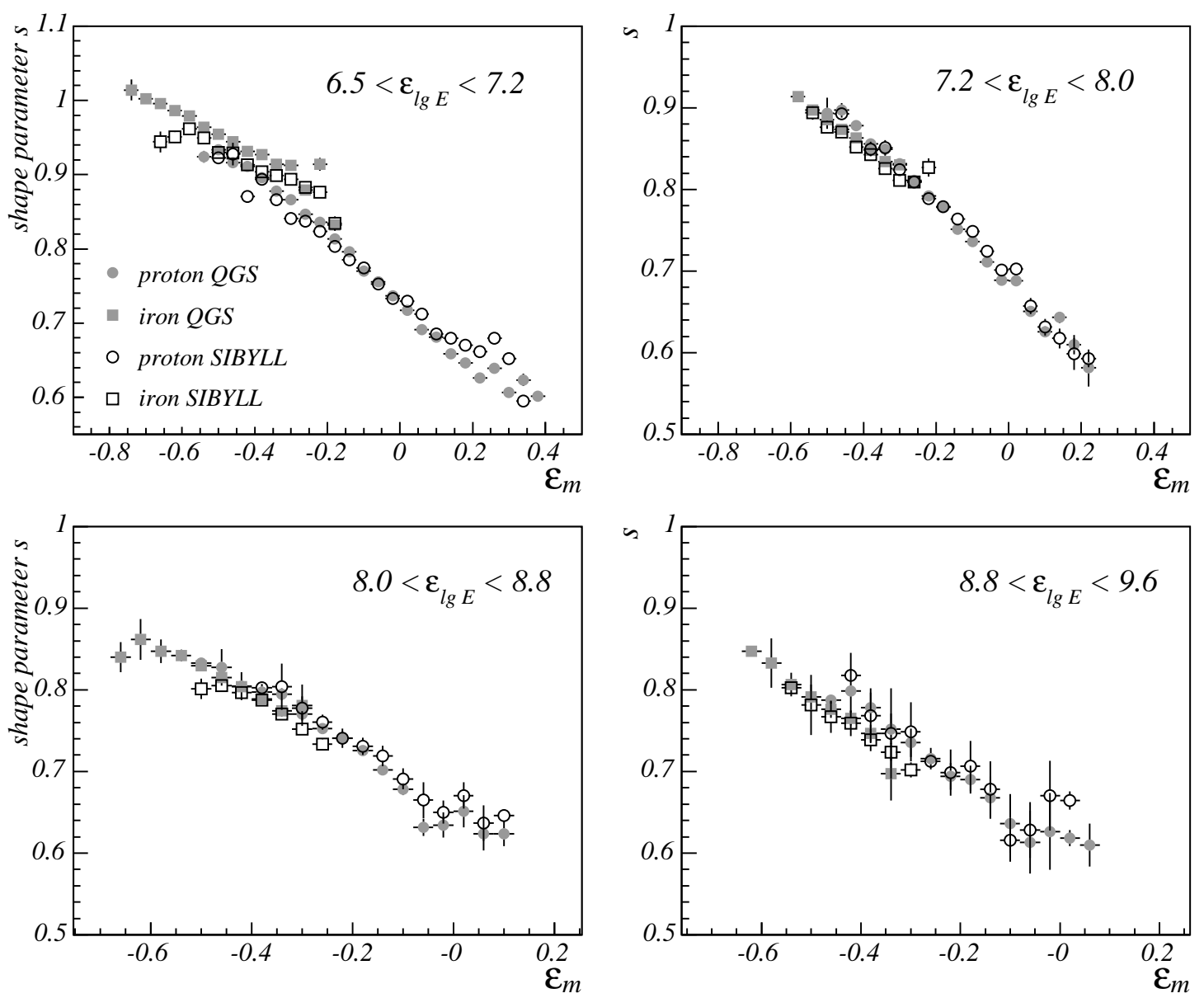

Fig. 12. Reconstructed shape parameter (of the modified NKG function) as a function of the mass estimator $\varepsilon_{m}$ for QGSJet and SIBYLL based simulations and different ranges of the energy estimator $\varepsilon_{\lg E}$. For reasons of clarity only two elemental masses are shown.

ues of the measured shape parameter however were found to disagree with the predictions of either of the two hadronic interaction models. While both models yield the same dependence of the average shower shape on $\lg N_{e}$ and $\lg N_{\mu}$, the absolute values appear smaller than the measured shape values for the whole considered range in $\lg N_{e}$ and $\lg N_{\mu}$.

Looking at the mean shape parameter as a function of primary energy, SIBYLL yields smaller values compared to QGSJet. The reason for this difference between the two models is a kind of "lighter" distribution of events in the $\lg N_{e}-\lg N_{\mu}-$ plane in case of SIBYLL: showers of same primary type and energy contain on average less muons but more electrons and therefore make up a smaller average value for the shape parameter compared to QGSJet. However, also the QGSJet predictions still underestimate the results from measurements by an almost constant amount of $\Delta s \sim 0.05$. 
Summarizing, both models are not able to describe the measured lateral distribution of the $e / \gamma$-component correctly. The details of the form of the lateral distribution depend on the hadronic interaction mechanism as well as on electromagnetic cascading processes. Thus, a variant of the QGSJet model that predicts a larger $e / \mu$-ratio, would give better consistency with data. However, the discrepancies might also be burried in the electromagnetic cascading algorithm EGS4 and its treatment of the multiple coulomb scattering process. Further improvements in the simulation models may be necessary, to understand and remove the discrepancies between data and simulations. Meanwhile, these results hopefully may help to stimulate this process and provide some additional hints.

\section{Acknowledgements}

The authors would like to thank the members of the engineering and technical staff of the KASCADE collaboration, who contributed to the success of the experiment with enthusiasm and committment. The KASCADE experiment is supported by the Ministry of Research of the Federal Government of Germany. The Polish group acknowledges support by KBN research grant 1 P03B 03926 for the years 2004-2006.

\section{References}

[1] Haungs, A., Rebel, H., Roth, M., Rep. Prog. Phys. 55 (2003) 1145

[2] Drescher, H.J., et al., Astroparticle Physics 21 (2004) 87

[3] Nishimura, J., in Prog. Cosmic ray Physics vol III, ed. J.G.Wilson (North Holland, Amsterdam, 1956)

[4] Kamata, K., \& Nishimura, J., in Prog. Theor. Phys. Suppl. 6 (1958) 93

[5] Greisen, K., in Prog. Cosmic ray Physics vol III, ed. J.G. Wilson (North Holland, Amsterdam, 1956)

[6] Antoni, T. et al. - KASCADE Collaboration, Nucl. Instr Meth. A 513 (2003) 490

[7] Antoni, T. et al. - KASCADE-Collaboration, Astropart. Phys. 14 (2001) 245

[8] Kalmykov, N.N. and Ostapchenko, S.S., Phys. Atom. Nucl. 56 (1993) 346

[9] Engel, R. et al., Proc. 26 ${ }^{\text {th }}$ Int. Cosmic Ray Conf. Salt Lake City (USA) 1 (1999) 415 
[10] Heck, D. et al., Report FZKA 6019, Forschungszentrum Karlsruhe (1998)

[11] Fassò, A. et al., FLUKA: Status and Prospective for Hadronic Applications, Proc. Monte Carlo 2000 Conf., Lisbon, Oct. 23-26, 2000, A. Kling et al. eds., Springer (Berlin) 955 (2001); www.fluka.org

[12] Nelson, W.R., Hirayama, H., and Rogers, D.W.O., Report SLAC 265, Stanford Linear Accelerator Center (1985)

[13] GEANT - Detector Description and Simulation Tool, CERN Program Library Long Writeup W5013, CERN (1993)

[14] Mayer, H.J., Nucl. Instr. Meth. A 311 (1992) 327

[15] Yoshida, S. et al., Phys. G: Nucl. Part. Phys. 20 (1994) 651

[16] Glushkov, A.V. et al., Proc. 25th ICRC vol 6 (1997) p. 233

[17] Coy, R.N. et al., Astropart. Phys. 6 (1997) 263

[18] Capdevielle, J.N., Cohen, J., J. Physics G: Nucl. Part. Phys. 31 (2005) 507

[19] Antoni, T. et al. - KASCADE-Collaboration, Astropart. Phys. 16 (2002) 373

[20] Weber, J.H. et al., Proc. $26^{\text {th }}$ Int. Cosmic Ray Conf. Salt Lake City (USA) 1 (1999) 415

[21] Antoni, T. et al. - KASCADE-Collaboration, Astropart. Phys. (2005) in press, preprint astro-ph/0505413 